\title{
Dimitri Karadimas ou la richesse de regards croisés entre l'Amazonie et la Mésoamérique
}

Guilhem Olivier

\section{(2) OpenEdition}

\section{Journals}

Édition électronique

URL : https://journals.openedition.org/jsa/15892

DOI : 10.4000/jsa.15892

ISSN : 1957-7842

Éditeur

Société des américanistes

\section{Édition imprimée}

Date de publication : 15 juin 2018

Pagination : 273-280

ISSN : 0037-9174

\section{Référence électronique}

Guilhem Olivier, « Dimitri Karadimas ou la richesse de regards croisés entre l'Amazonie et la

Mésoamérique », Journal de la Société des américanistes [En ligne], 104-1 | 2018, mis en ligne le 15 juin 2018, consulté le 08 septembre 2022. URL : http://journals.openedition.org/jsa/15892 ; DOI : https:// doi.org/10.4000/jsa.15892 


\title{
Dimitri Karadimas ou la richesse de regards croisés entre l'Amazonie et la Mésoamérique
}

\author{
Guilhem OLIVIER *
}

Foisonnante, l'œuvre de Dimitri Karadimas non seulement renouvelle les études sur l'histoire et l'anthropologie de l'aire amazonienne, mais aussi consolide et enrichit un dialogue amorcé il y a quelques années entre certains spécialistes de cette zone géographique et d'autres qui étudient la Mésoamérique.

Ainsi, nos recherches sur la chasse en Mésoamérique nous ont conduit à des lectures diverses sur des pratiques cynégétiques dans d'autres régions du monde, en Sibérie, en Amérique du Nord et bien sûr en Amazonie. À la différence de nos discrets chasseurs aztèques ou mayas dont les coutumes ne font l'objet que de brèves gloses dans les chroniques anciennes, nous découvrions avec émerveillement des chasseurs prolixes qui expliquaient de façon détaillée leur « vision du monde ». Ces témoignages coïncidaient souvent et surtout complétaient les données fragmentaires recueillies en Mésoamérique. Au détour d'une lecture, nous découvrions que le Maître des animaux chez les Miraña était une guêpe ou encore que le concept de prédation était au cœur des rites initiatiques de ce peuple, éléments qui entraient en résonance avec mes propres recherches.

La lecture de La raison du corps. Idéologie du corps et représentation de l'environnement chez les Miraña d'Amazonie colombienne (Karadimas 2005), de divers articles et chapitres de livres et surtout de l'impressionnant manuscrit intitulé Dieux, guerriers, parasites célestes. Perceptions, mythes et images en Amérique du Sud ${ }^{1}$, ne saurait laisser indifférent un chercheur confronté à des problématiques similaires dans une aire géographique éloignée, mais culturellement proche à bien des égards. Inspiré par les œuvres de Claude LéviStrauss et de Philippe Descola, le parcours intellectuel de Dimitri Karadimas part de l'analyse du corps et de la représentation de l'environnement chez les Miraña, et va jusqu'à des analyses comparées entre les mythes actuels et les

* Instituto de Investigaciones Históricas, Universidad Nacional Autónoma de México [olivier@unam.mx].

1. Présenté par Dimitri Karadimas lors de la soutenance de son Habilitation à diriger des recherches (HDR) au Collège de France le 3 juin 2016. 
arts anciens de l'Amérique du Sud. Nous savons que dans les Mythologiques Claude Lévi-Strauss $(1964,1966,1968,1971)$ n'abordait qu'incidemment les données anciennes mésoaméricaines et andines, en raison, expliquait-il, du caractère « lettré » des sources concernant ces civilisations. Dans Dieux, guerriers, parasites célestes, Karadimas revient sur cette posture de Lévi-Strauss et démontre brillamment comment les mythes actuels des Miraña et d'autres peuples voisins peuvent contribuer à élucider la signification de multiples objets archéologiques, non seulement de l'ancienne Colombie, mais aussi des hautes civilisations andines.

Dans l'impossibilité de commenter de façon détaillée l'ensemble de l'œuvre de Dimitri Karadimas, nous avons choisi de nous attacher à certains aspects de ses travaux susceptibles de faire écho à des conceptions semblables en Mésoamérique, un peu à l'image de ce masque que les Miraña appellent le « répétiteur », lequel représente justement l'écho perçu dans la forêt.

Ainsi, dans Dieux, guerriers, parasites célestes, nous avons été frappé par les analyses concernant les identifications stellaires de divers personnages : héros mythiques, chefs et ennemis chez les Miraña et leurs voisins, ou encore acteurs des anciens rituels tupinambas. Karadimas étudie avec finesse comment ces personnages sont identifiés au soleil, à la lune et/ou aux étoiles, notamment Orion, avec parfois l'apparition d'intéressants processus d'inversion selon les rituels, par exemple, lors du décès du chef. Ces phénomènes évoquent, dans leur complexité même, les associations astronomiques si fréquentes dans les registres iconographiques, mythiques et rituels mésoaméricains. Au-delà de l'abus des lectures astronomiques en vogue chez les mexicanistes de la fin du XIX siècle et du début du $\mathrm{Xx}^{\mathrm{e}}$ siècle, il est indéniable que le « code astronomique » était fondamental en Mésoamérique précolombienne. Une partie des critiques à cette approche était fondée précisément sur la diversité des associations astronomiques que certains chercheurs attribuaient aux divinités : le savant allemand Eduard Seler, par exemple, présentait Tezcatlipoca, le « Seigneur au miroir fumant», comme une divinité solaire puis, dans un autre écrit, rectifiait sa position en disant qu'il s'agissait d'un dieu lunaire ou encore d'un numen associé à Vénus.

À l'instar de Karadimas qui insiste sur l'importance du contexte qui préside aux identifications astronomiques, nous avons proposé que les identifications de Tezcatlipoca au soleil, à la lune ou à Venus, loin d'être contradictoires, obéissaient à la position de cette divinité dans des cycles cosmogoniques durant lesquels il alternait avec son jumeau divin Quetzalcoatl (Olivier 1997). Quant au personnage du mythe kaliña qui perd une jambe et devient Orion dont Karadimas propose une analyse, il rappelle à certains égards le dieu Tezcatlipoca, amputé lui aussi, qui se transforme en la Grande Ourse. D'autre part, le lien établi par l'auteur entre les taches sur le visage d'un personnage et l'inceste à partir de mythes que l'on retrouve aussi en Mésoamérique, est très suggestif. Ce symbolisme s'étendrait aux taches du jaguar associé à la lune et à son comportement 
incestueux. En Mésoamérique, le grand félin est également associé à l'astre sélénite, quoique généralement crédité d'une sexualité modérée ; aussi est-il recommandé aux veufs de consommer de la chair de jaguar pour atténuer leurs désirs sexuels dus à la perte de leurs épouses. Ajoutons que les taches du pelage du grand félin sont identifiées à des étoiles par les Mayas et les Totonaques.

Plus avant dans le même manuscrit, Karadimas mentionne l'utilisation divinatoire d'araignées - de mygales plus précisément - et l'association entre les défunts et les mouches ou plus probablement les guêpes (p. 371-372). Une fois encore, l'auteur éclaire d'un jour nouveau les données anciennes et modernes à partir de sources iconographiques et écrites et propose que « le modèle général reste cependant celui des guêpes parasitoïdes et de leur mode de reproduction grâce à d'autres insectes, dans lequel les humains occupent la place de la victime-proie alors que la guêpe celle des dieux prédateurs, raison pour laquelle, durant la période des chroniques, se déroule la pratique de l'arachnomancie » (p. 373). Dans l'ancien Mexique, on utilisait également des insectes à des fins divinatoires, par exemple en interprétant les mouvements d'un scarabée (le pinahuiztli) sur un cosmogramme tracé sur le sol.

Quant aux araignées représentées dans l'iconographie moché, Karadimas les interprète comme des vaincus, quand bien même elles apparaissent sur un pan de mur de la Huaca de la Luna portant « une tête coupée à bout de bras » (p. 381). Les têtes décapitées transportées dans un filet représenteraient les œufs d'arthropodes et l'auteur rappelle, sur la base des travaux d'Anne-Christine Taylor, le symbolisme des têtes réduites chez les Jivaro-Shuar, lesquelles représentent les identités futures à naître dans le groupe des meurtriers, c'est-à-dire les futurs fils des femmes du groupe vainqueur. Cela étant, ne pourrait-on pas évoquer à propos de ces images la réversibilité du statut de meurtrier et les processus fréquents en Amazonie et ailleurs d'identification entre le chasseur ou le guerrier et sa proie victime ? Certaines images d'araignées pourraient effectivement les représenter sous la forme d'un redoutable prédateur - ce qu'elles sont sans doute - tandis que d'autres les figureraient comme proies de prédilection des guêpes. Karadimas considère que certaines images moché d'insectes possédant des traits à la fois de guêpe et d'araignées « exprimeraient graphiquement une transformation qui semble se réaliser réellement de l'arachnide vers l'hyménoptère » (p. 392). Ne pourrait-on pas aussi imaginer que le «mélange » de ces deux insectes manifeste leurs caractéristiques communes, probablement prédatrices ? On songe à un animal étrange nommé mazamiztli, «puma-cerf»-décrit par les collaborateurs nahuas du franciscain Bernardino de Sahagún - qui se distingue par sa capacité à se glisser sans être reconnu parmi les cervidés pour ensuite les éventrer avec ses griffes et dévorer leurs entrailles. Ce cerf carnivore n'est pas sans évoquer un récit à la fois cocasse et révélateur inclus dans le manuscrit de Huarochiri, qui explique comment, suite à un lapsus, les cerfs cannibales furent transformés en prises de chasse. Nous revenons ainsi au thème de la réversibilité du statut 
des chasseurs et des chassés. Reste à savoir si ces dynamiques d'identification peuvent se combiner avec ce que Karadimas appelle « une conception générale d'un modèle que nous avons qualifié de "métamorphose prédatrice" » (p. 497). Ses analyses des rites d'initiation le laissent penser, au moins du côté des jeunes gens, puisque ces derniers « se voient identifiés à la guêpe après avoir été assimilés à la proie (chenille, chrysalide, etc.), alors que les jeunes filles ka'apor sont maintenues dans l'identification avec les araignées, c'est-à-dire dans leur futur rôle de mère » (p. 450).

Toujours à l'affût de possibles éléments communs entre l'Amazonie et la Mésoamérique, nous avons été frappé par la figure miraña du Maître des animaux analysée minutieusement par Karadimas. Malgré l'absence dans nos matériaux mésoaméricains d'identification explicite de cette figure mythologique avec une guêpe parasitoïde, il existe cependant quelques données fragmentaires qui attestent des liens du Maître des animaux avec les abeilles et les guêpes. Par exemple, dans les années 1930, les Mayas du Yucatan attribuaient la protection des cervidés à Zip, un petit cerf portant un nid de guêpes entre ses bois. Sifflant entre ses sabots pour alerter ses congénères de l'approche des chasseurs - c'est pourquoi il existe des invocations spéciales pour solliciter sa discrétion - Zip peut tuer un chasseur qui a osé pointer son arme sur lui en envoyant une guêpe le piquer. À la même époque, les Yaquis du Sonora vénèrent également un petit cerf nommé Malici, doté lui aussi d'un nid de guêpes dans sa ramure, tandis que le Maître des animaux anthropomorphe des Chinantèques d'Oaxaca apparaît portant un essaim d'abeilles sur le dos.

Au sujet des aspects prédateurs, voire cannibales, de cette figure surnaturelle mis en évidence par Karadimas, ils coïncident avec bien des traits des Maîtres des animaux en Mésoamérique dont le prototype ancien n'est autre que Tezcatlipoca sous la forme d'un jaguar, tecuani, le «mangeur de personne ». Chez les Miraña, le Maître des animaux agit à l'image d'une guêpe et se confond avec elle, " "inséminant" les humains pour reproduire le gibier [...]. Les humains sont ainsi les araignées du Maître des animaux, où ses proies » (p. 458). Les Nahuas de la Sierra Negra de Puebla décrivent comment le Maître des animaux conserve des têtes humaines décapitées suspendues aux poutres de sa maison, tandis que les humains en font de même avec les crânes des animaux chassés.

Dans le prolongement de ses travaux sur l'iconographie ancienne de l'Amérique du Sud, Karadimas a abordé l'art mésoaméricain dans un article à la fois novateur et provocateur ayant pour objet l'une des figures les plus importantes du panthéon mésoaméricain, le célèbre Serpent à plumes ${ }^{2}$. L'auteur propose de façon originale que « la figure mythologique du serpent à plumes qui donnera naissance au Quetzalcoatl du Postclassique se construit à partir d'une chenille

2. Karadimas 2014. Dans un autre article récent, Dimitri Karadimas (2016) traite de la figure mythologique de Tlaloc, le dieu de la pluie. 
hautement urticante d'un papillon de nuit, Automeris io qui sous sa forme adulte, offre un mimétisme construit sur l'image d'une face de chouette ou de jaguar, ces derniers animaux se retrouvant combinés dans des artefacts à forme de papillon ». À partir de ces prémices, on peut compléter certaines intuitions de Karadimas, par exemple au sujet d'un serpent à deux têtes qu'il identifie à une chenille « arpenteuse » nommée tlatamachihauni en nahuatl.

Or ce serpent à deux têtes apparaît aussi sous le nom de maquizcoatl, « serpent bracelet ", qui correspond à l'un des noms de Huitzilopochtli, "Colibri Gaucher », la divinité tutélaire des Mexicas. Il convient d'ajouter que l'un des doubles animal de Huitzilopochtli est précisément un xiuhcoatl, un serpent de feu identifié par Karadimas à une chenille. Enfin, l'association que l'auteur établit entre des chenilles terrifiantes - leurs piqures provoquent des saignements létaux - et des chenilles excentriques en obsidienne en forme de serpent est basée sur l'utilisation d'objets en obsidienne à l'occasion de pratiques rituelles d'autosacrifices durant lesquels on pratiquait des saignées en diverses parties du corps. Pour renforcer cette hypothèse, signalons que les serpents apparaissent souvent dans l'iconographie mésoaméricaine pour figurer des flots de sang, par exemple dans des scènes de décapitation.

Pour finir, les travaux de Dimitri Karadimas peuvent contribuer à l'interprétation d'une scène particulièrement énigmatique du Codex Borgia, magnifique manuscrit précolombien dont les planches centrales suscitent encore de nombreuses interrogations. Décrivons brièvement la scène complexe peinte sur la planche 44 : la déesse Xochiquetzal y est allongée et de son cœur surgit un arbre au tronc multicolore chargé de fleurs ; au-dessus de l'arbre, se tient Quetzalcoatl sous un déguisement de colibri et un personnage de chauve-souris duquel coule un flot de sang qui atteint la déesse. Cette scène a été interprétée comme illustrant un mythe d'origine des menstruations, lesquelles furent déclenchées par la morsure d'une chauve-souris infligée à Xochiquetzal, la déesse des fleurs. Or l'association entre la chauve-souris et les règles féminines se retrouve chez les Kogi de Colombie, les femmes de ce groupe se demandant entre elles « Est-ce que la chauve-souris t'a mordue ? » pour savoir si elles sont indisposées. Quant aux Miraña étudiés par Karadimas, ils affirment qu'il est nécessaire que les femmes « aient leur chauve-souris », c'est-à-dire leurs règles, pour pouvoir tomber enceinte. Revenons au Codex Borgia : la scène centrale est entourée d'un cadre formé par les parois d'édifices surmontées de couteaux de silex et de fleurs associées à des rites d'intronisation. À l'entrée de ces édifices apparaissent trois futurs gouvernants dont les têtes émergent du corps de serpents de feu; en outre, leurs nez sont mordus respectivement par un jaguar, un quetzal et un aigle, animaux associés à la guerre et au pouvoir des dirigeants (Figure 1, page suivante).

Dans un livre récent, nous avons proposé que les rituels initiatiques durant lesquels on perçait la cloison nasale des gouvernants fussent équivalents aux 


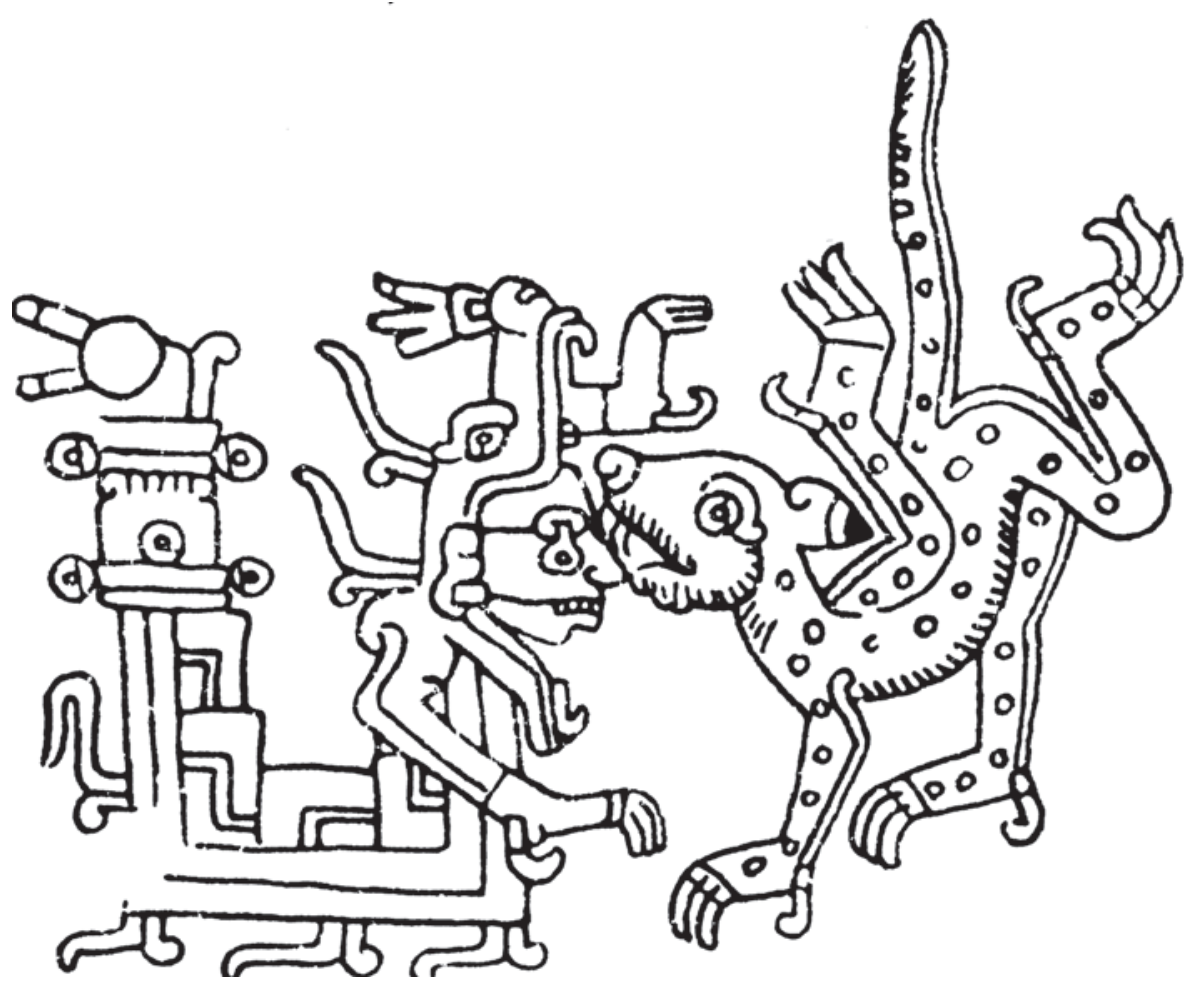

Fig. 1 - Códice Borgia, Eduard Seler (ed.), Fondo de Cultura Económica, México, 1963, pl. 44 ; original conservé à la Bibliotèque du Vatican (dessin de Rodolfo Ávila).

rites liés à la première menstruation des femmes dont le mythe d'origine est illustré sur cette planche (Olivier 2015). La perforation de la cloison nasale représente le sacrifice symbolique des futurs gouvernants, lesquels sont assimilés à des prises de chasse immolées. Or en livrant dans La raison du corps une interprétation des statues en or de l'ancienne culture taïrona de Colombie affublées d'ornement de nez en forme de chauve-souris, Karadimas propose que la présence d'une chauve-souris à hauteur de la bouche transforme cet organe en une matrice capable de générer des paroles à offrir au Maître des animaux en échange de prises de chasse. Ce n'est pas tout. Lorsqu'il analyse le rituel initiatique de Yurupari durant lequel les adolescents sont fouettés, l'auteur considère que «si les trompes de Yurupari sont évoquées comme un équivalent de l'accession à la faculté de reproduction sur le modèle des saignements menstruels, il faudrait que les hommes subissent un saignement équivalent » (p. 274). En outre, Karadimas propose que les initiés, assimilés à des chenilles-larves, subissent plusieurs changements de peau avant d'aborder le 
processus de métamorphose. Il va sans dire qu'à la lecture de ces interprétations concernant des matériaux sud-américains, la présence des initiés du Codex Borgia à l'intérieur du corps des serpents de feu - identifiés à des chenilles, rappelons-le - acquiert une nouvelle signification.

Ces parallélismes que l'on peut observer entre certains rites d'initiation mésoaméricains et amazoniens sont remarquables dans la mesure où ils contribuent parfois, au-delà de conceptions partagées, à expliquer des aspects obscurs de l'iconographie et des témoignages écrits de l'ancien Mexique. De même, il conviendrait d'évaluer à l'aune de matériaux mésoaméricains la viabilité des réflexions de Karadimas sur les rapports entre mythe et iconographie : « La variation structurale dégagée par Lévi-Strauss quant aux structures des mythes est ainsi également valide dans l'analyse des pluralités d'expressions iconographiques américaines tant que l'animal fantastique, une fois rencontré, est regardé pour ce qu'il est : une retranscription analogique et anthropomorphe d'un animal réel qui peut jouer le rôle d'un exemplaire supplémentaire dans les variantes combinatoires » (p. 399).

C'est dire la richesse de l'œuvre de Dimitri Karadimas qui constitue une source d'inspiration originale pour tous ceux qui souhaitent dépasser les frontières de spécialités trop étroites et osent s'aventurer sur des chemins comparatifs plus complexes, par exemple, entre l'Amazonie et la Mésoamérique.

\section{Références citées}

KARADIMAs Dimitri

2005 La raison du corps. Idéologie du corps et représentation de l'environnement chez les Miraña d'Amazonie colombienne, Peeters (Langues et sociétés d'Amérique traditionnelle, 9), Paris.

2014 «Voir une chenille, dessiner un serpent à plumes. Une relecture analogique de l'hybridité et des êtres imaginaires en Mésoamérique préhispanique ", Journal de la Société des américanistes, 100 (1), p. 7-43.

2016 «Caracóis emplumados e Tlaloc na Mesoamérica pré-hispânica : abordagem comparativa com o noroeste amazônico », Revista de Antropologia, 59 (1), p. 108-144.

LÉVI-STRAuss Claude

1964 Le cru et le cuit, Plon, Paris.

1966 Du miel aux cendres, Plon, Paris.

1968 L'origine des manières de table, Plon, Paris.

1971 L'homme nu, Plon, Paris.

OLIVIER Guilhem

1997 Moqueries et métamorphoses d'un dieu aztèque : Tezcatlipoca, le « Seigneur au miroir fumant », Institut d'ethnologie du musée de l'Homme/Centre d'études mexicaines et centraméricaines, Paris. 
Guilhem OLIVIER

OLIVIER Guilhem

2015 Cacería, sacrificio y poder en Mesoamérica. Tras las huellas de Mixcóatl, "Serpiente de Nube", Instituto de Investigaciones Históricas, Universidad Nacional Autónoma de México/Fondo de Cultura Económica/Fideicomiso Felipe Teixidor y Montserrat Alfau de Teixidor/Centro de Estudios Mexicanos y Centroamericanos, México. 